\title{
Magnetic resonance myocardial perfusion imaging - still new or a routinely used tool in coronary artery disease diagnostics?
}

\author{
tukasz A. Małek \\ Department of Interventional Cardiology and Angiology, Magnetic Resonance Unit, Institute of Cardiology, Warsaw, Poland
}

Postep Kard Inter 2012; 8, 3 (29): 225-228

DOI: 10.5114/pwki.2012.30402

\begin{abstract}
The article presents the newest data on magnetic resonance myocardial perfusion imaging in the diagnostics of coronary artery disease. Studies completed in recent years demonstrate that this method is superior to some other routinely used non-invasive techniques of myocardial ischemia assessment. Non-invasive coronary artery diagnostics consisting of anatomic assessment by means of computed tomography and functional assessment by means of cardiovascular magnetic resonance reduce the frequency of required coronary angiographies and hospitalizations related to them and therefore lowers the risk of invasive diagnostics and costs of treatment.
\end{abstract}

Key words: stable angina, cardiovascular magnetic resonance, myocardial ischemia, perfusion imaging

\section{Introduction}

According to current guidelines for clinical practice coronary revascularization aimed at reduction of symptoms or improvement of prognosis may be performed without additional examinations in patients with $>50 \%$ coronary artery stenosis accompanied by angina or its equivalents which cannot be controlled by means of optimal pharmacotherapy and in patients with severe/critical stenosis of the coronary artery (>90\% of the diameter) [1]. In all other situations additional functional testing to assess myocardial ischemia is required. The most frequently observed situations include: 1) the presence of significant, but not severe/critical stenosis of the coronary artery in asymptomatic patients or in patients with atypical symptoms, 2) the presence of borderline diameter stenosis (approx. 50-70\%) regardless of the presence of symptoms, 3) the presence of multi-vessel coronary artery disease regardless of symptoms where additional assessment is necessary to plan the type and extent of revascularization.

In all of the presented situations noninvasive testing for myocardial ischemia should be considered as the first choice. Invasive diagnostics by means of fractional flow reserve (FFR) should be reserved mainly for patients in whom noninvasive tests are contraindicated or unobtainable, or the results of those tests are not diagnostic, as well as for patients subjected to percutaneous coronary intervention/coronary angiography for other reasons.

According to clinical practice guidelines published 2 years ago, the noninvasive methods having the highest class of recommendations (class I) for the detection of myocardial ischemia are stress echo and single-photon emission computed tomography (SPECT). These recommendations were based on the highest experience with the use of these methods measured also with the number and quality of publications including reports showing the relation between detection of myocardial ischemia and patient's prognosis. Magnetic resonance myocardial perfusion imaging (MRMPI) and positron emission tomography (PET) obtained class II of recommendations. It is worth noting that class II of recommendations means that the weight of evidence/opinion is in favor of usefulness/efficacy of the assessed method of management [2, 3].

Several large studies in the field of MRMPI have been presented since the publication of the cited guidelines. The results are discussed below. They strengthen the position of this method in the functional diagnostics of coronary artery disease and will most likely cause an increase of the class of recommendations for this examination in the future guidelines.

\section{Corresponding author:}

Łukasz A. Małek MD, PhD, Department of Interventional Cardiology and Angiology, Magnetic Resonance Unit, Institute of Cardiology, 42 Alpejska, 04-628 Warsaw, Poland, tel.: +48 22343 46 57, fax: +48 2261338 19, e-mail: Imalek@ikard.pl

Praca wpłynęła: 27.07.2012, przyjęta do druku: 23.08.2012. 


\section{Method description}

The study is based on the assessment of the presence of subendocardial perfusion defects observed during the first pass of gadolinium-based contrast medium through the myocardium at peak hyperemia stimulated by adenosine $(0.14 \mathrm{mg} / \mathrm{kg} / \mathrm{min}$ i.v. for $3 \mathrm{~min}$, short-lasting effect) or dipyridamole infusion $(0.57 \mathrm{mg} / \mathrm{kg}$ i.v. for $4 \mathrm{~min}$, longer-lasting effect). A new drug that may become useful in the near future is regadenoson, administered in a $0.4 \mathrm{mg}$ i.v. bolus and leading to fast occurrence of hyperemia and its persistence for a longer time in comparison to adenosine (half-life time of 2-3 min in comparison to $30 \mathrm{~s}$ for adenosine). The test may be supplemented by the assessment of the first pass of gadolinium-based contrast medium through the myocardium at rest (before drug administration or after cessation of its effect), but it is not mandatory. In routine practice the analysis of perfusion defects is usually performed visually in each of the 17 segments of the left ventricle defined by the American Heart Association (AHA), but methods of quantitative analysis also exist. The latter are more laborious and require additional software, which together with the fact that these methods were not shown to have higher diagnostic accuracy makes them less clinically useful. It should be noted that visual assessment is sufficient to determine whether the extent of ischemia exceeds $10 \%$ of the left ventricular mass, which often impacts the decision to perform coronary revascularization [4].

Advantages of MRMPI include the possibility of simultaneous assessment of myocardial contractility and myocardial scars after previous myocardial infarctions (also silent), which may have an additional diagnostic significance.

To allow diagnostic assessment patients should refrain from intake of beverages and food including caffeine, $\beta$-blockers, calcium channel blockers, nitrates and dipyridamole/theophylline for $24 \mathrm{~h}$ before the examination.

Cardiovascular magnetic resonance is not free from limitations. Patients cannot have some metallic foreign objects in the body (such as a pacemaker, implanted cardioverterdefibrillator, or clips after brain aneurysm surgery) and the glomerular filtration rate should exceed $30 \mathrm{ml} / \mathrm{min}$. Gadolinium-based contrast media should not be administered to patients with end-stage renal failure due to the risk of nephrogenic systemic fibrosis. Registration of optimal images may be impossible in case of atrial fibrillation or frequent extrasystoles. Other limitations are limited to MRMPI and include contraindications to adenosine or dipyridamole administration such as hypersensitivity to these medications, $2^{\text {nd }}$ or $3^{\text {rd }}$ degree atrioventricular block, sick sinus syndrome, long QT syndrome, severe hypotension or the risk of bronchospasm (mainly due to severe asthma).

\section{Recent clinical research}

MR-IMPACT was the first randomized study which on a large scale (18 centers in Europe and USA, different scan- ners with field strength of $1.5 \mathrm{~T}$ ) analyzed the efficacy of MRMPI (with adenosine infusion) in comparison to SPECT in the detection of coronary artery disease verified with coronary angiography [5]. The study was also set to assess the optimal dose of gadolinium-based contrast medium used for the assessment of perfusion. It was found to be $0.1 \mathrm{mmol} / \mathrm{kg}$. In patients who received an optimal dose of gadolinium-based contrast medium MRMPI had similar efficacy to SPECT. However, the analysis of all patients randomized into the study showed superiority of MRMPI over SPECT. This was the starting point for the execution of the large, multicenter randomized study MR-IMPACT II (33 centers in Europe and USA), the results of which were announced this year [6].

The MR-IMPACT II study demonstrated that perfusion assessment by means of magnetic resonance had higher sensitivity and lower specificity in detection of > 50\% diameter stenosis on X-ray coronary angiography in comparison to SPECT (both gated and ungated). The superiority of MRMPI in terms of sensitivity was also noted in patients with multi-vessel coronary artery disease and in the female and male populations analyzed separately.

The results of this study correspond to the results of the largest single center randomized study, CE-MARC, which was published at the beginning of this year [7]. The study compared the multi-parametric magnetic resonance protocol (including left ventricular systolic function assessment, MRMPI at rest and after administration of adenosine, coronary magnetic resonance angiography and late gadolinium enhancement) with SPECT to detect significant coronary artery stenosis. MRMPI had higher sensitivity (86.5\% vs. $66.5 \%)$ and similar specificity ( $83.4 \%$ vs. $82.6 \%)$ to SPECT. The MRMPI was able to detect smaller areas of ischemia in comparison to SPECT, which is related mainly to the better spatial resolution of magnetic resonance.

The key element which strengthens the position of a diagnostic test is the evidence of a relation between abnormalities observed with that test and the patient's prognosis. Until recently only single reports of this type were available for MRMPI [8]. For this reason it is worth mentioning the article by Coelho-Filho et al. published a year ago, which demonstrated the value of ischemia observed with MRMPI in the prediction of clinical events (cardiovascular death, myocardial infarction) [9].

In the era of searching for different means to limit the rising costs of medical care, a cost-analysis of inclusion of MRMPI in the diagnostic model of coronary artery disease was performed. The cost of MRMPI (including cost of control examinations) was compared to a strategy of direct referral for coronary angiography in 2717 patients from the European registry of cardiovascular magnetic resonance studies (EuroCMR Registry) [10]. The new diagnostic scheme led to a $46-50 \%$ reduction of costs in the case of hospitalized patients and to a $23-50 \%$ reduction of costs in the case of outpatient coronary angiographies. 
The results were most likely driven by the fact that myocardial ischemia was detected in only $21 \%$ of patients with suspected coronary artery disease. This suggests that it is worth performing MRMPI before coronary angiography and to limit access to coronary angiography to patients with confirmed presence of myocardial ischemia and/or postinfarction scar (assessed simultaneously by means of late gadolinium enhancement).

In another study the use of magnetic resonance decreased the need for coronary angiography by over $62 \%$ and led to reduction of diagnostic costs, especially in lowrisk patients [11]. The cost reduction was not observed only in patients at the highest risk. The authors of the study conclude that because of the very high negative predictive value of MRMPI, which means that the lack of perfusion defects suggests with a high probability that there are no clinically significant lesions in coronary arteries, the study may become a gatekeeper to coronary angiography, which is often ordered too easily.

The discussion presented above is supplemented in an interesting way by the results of another study analyzing execution of computed tomography coronary angiography (CTCA) followed by MRMPI in the diagnosis of coronary artery disease $[12,13]$. Both noninvasive tests were successfully performed in over $90 \%$ of patients. No perfusion defects were detected in over $90 \%$ of patients without changes in the coronary arteries on CTCA and in over 80\% with non-significant changes in coronary arteries on CTCA, which shows that CTCA is an effective method to exclude the presence of clinically significant coronary artery disease. On the other hand, perfusion defects on MRMPI were observed in only $40 \%$ of patients with significant lesions on CTCA, which confirms the limitation of an anatomically based approach to diagnosis of coronary artery disease. Only the combination of both methods allows a comprehensive, noninvasive assessment of patients with suspected coronary artery disease. Based on this approach, a new algorithm of coronary artery disease diagnosis may be proposed (Figure 1).

Studies aimed to further strengthen the position of MRMPI in the diagnosis of coronary artery disease are ongoing. The MR-INFORM study uses FFR as a reference for MRMPI, similarly to the CE-MARC II study, which is based only on scanners with a field strength of 3T. The use of cardiovascular magnetic resonance (along with SPECT and echocardiography) is also an element of the ISCHEMIA study, which is aimed at finding an optimal management strategy (invasive approach vs. medical treatment only) for patients with at least moderate myocardial ischemia. Further improvement of the quality of MRMPI studies may be possible with wider introduction of high-sensitivity methods of magnetic resonance perfusion detection [14].

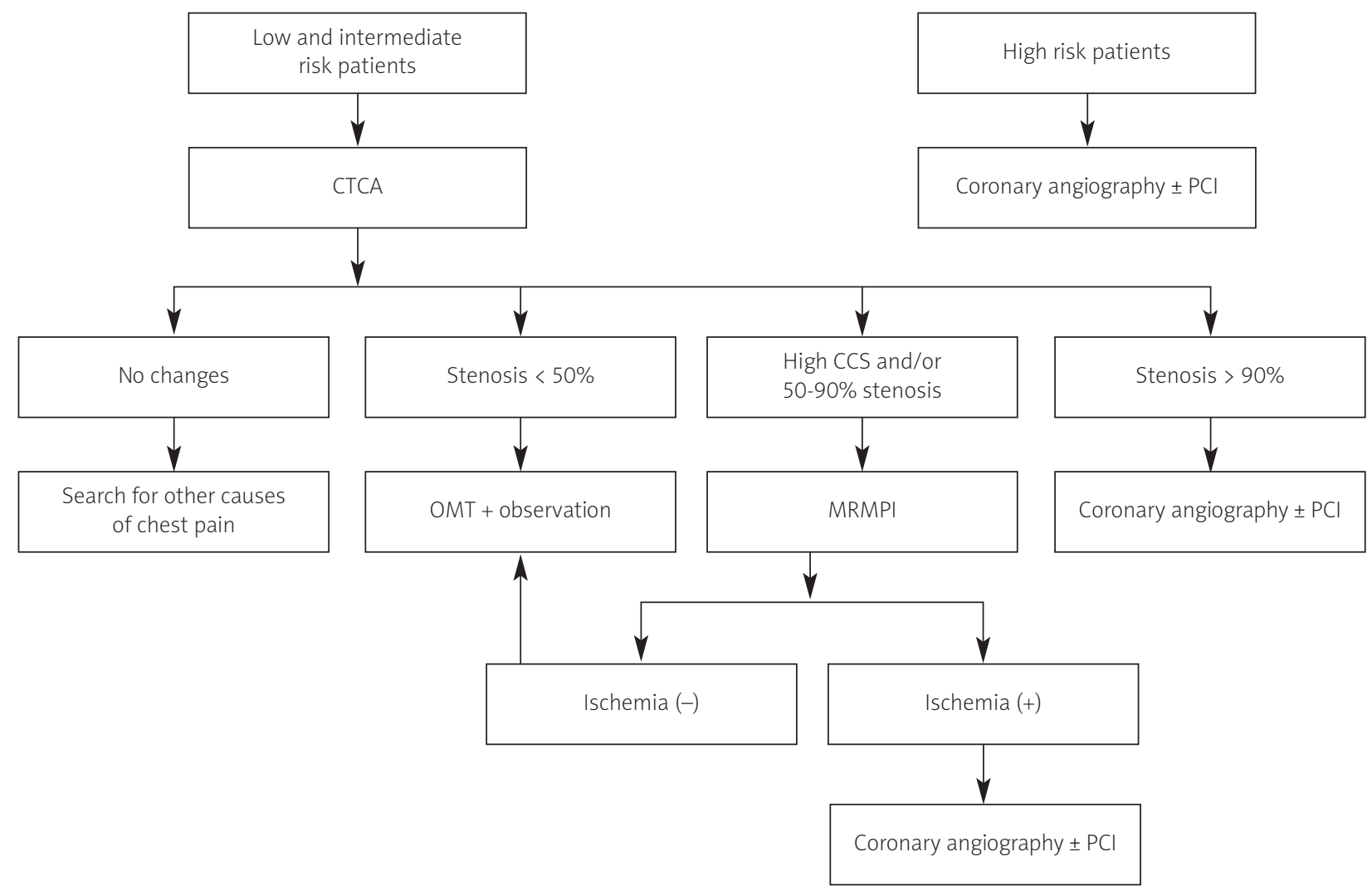

Fig. 1. The algorithm proposed for thecoronary artery disease diagnostics

MRMPI - magnetic resonance myocardial perfusion imaging, OMT - optimal medical treatment, CTCA - computed tomography coronary angiography, CCS - coronary calcium score, $\mathrm{PCl}$ - percutaneous coronary intervention 


\section{Summary}

Successful results of the presented studies and an increasing frequency of performed MRMPI tests in the reference centers suggests that this method may soon become a routinely used tool for the functional assessment of coronary artery disease. Other factors which encourage the use of this test include its safety (lack of exposure to ionizing radiation, lack of the need for administration of catecholamines, extremely rare allergic reactions to gadolinium-based contrast media), short time of examination (approximately $30 \mathrm{~min}$ in experienced centers) and increasing availability of this study not only in public, but also in private centers [15].

The combination of anatomic assessment by means of computed tomography coronary angiography and functional assessment by means of cardiovascular magnetic resonance (including mainly the analysis of perfusion during hyperemia, but also myocardial contractility and late gadolinium enhancement) may in the next years become a method of choice in most patients with suspected coronary artery disease. This approach will allow the frequency of invasive examinations to be limited.

Noninvasive tests performed before coronary angiography create a possibility to gather information helpful in planning the percutaneous coronary intervention (PCI). This information includes assessment of the lesion anatomy with computed tomography, which may improve the choice of the right stent or localization of the artery responsible for ischemia (culprit vessel) on cardiovascular magnetic resonance and/or the assessment of myocardial viability of the area supplied by the narrowed coronary artery. The new model of diagnostics may also help to choose other equipment (such as the type of guidewire or guiding catheter) necessary for the optimal execution of a treatment procedure during single cardiac catheterization. Data obtained that way should increase the frequency of percutaneous coronary interventions performed directly after coronary angiography (ad hoc). Comprehensive noninvasive diagnostics before coronary angiography should also permit the introduction of antiplatelet drugs only in patients qualified for ad hoc $\mathrm{PCl}$.

\section{References}

1. Wijns W, Kolh P, Danchin N, et al. Guidelines on myocardial revascularization. Eur Heart J 2010; 31: 2501-2555.

2. Nandalur KR, Dwamena BA, Choudhri AF, et al. Diagnostic performance of stress cardiac magnetic resonance imaging in the detection of coronary artery disease: a meta-analysis. J Am Coll Cardiol 2007; 50: 1343-1353.

3. Costa MA, Shoemaker S, Futamatsu H, et al. Quantitative magnetic resonance perfusion imaging detects anatomic and physiologic coronary artery disease as measured by coronary angiography and fractional flow reserve. J Am Coll Cardiol 2007; 50: 514-522.

4. Hachamovitch R, Hayes SW, Friedman JD, et al. Comparison of the short-term survival benefit associated with revascularization compared with medical therapy in patients with no prior coronary artery disease undergoing stress myocardial perfusion single photon emission computed tomography. Circulation 2003; 107: 2900-2907.

5. Schwitter J, Wacker CM, Van Rossum AC, et al. MR-IMPACT: Comparison of perfusion-cardiac magnetic resonance with singlephoton emission computed tomography for the detection of coronary artery disease in a multicentre, multivendor, randomized trial. Eur Heart J 2008; 29: 480-489.

6. Schwitter J, Wacker CM, Wilke N, et al. MR-IMPACT II: Magnetic resonance imaging for myocardial perfusion assessment in coronary artery disease trial: perfusion-cardiac magnetic resonance vs. single-photon emission computed tomography for the detection of coronary artery disease: a comparative multicentre, multivendor trial. Eur Heart J 2012, Mar 4.

7. Greenwood JP, Maredia N, Younger JF, et al. Cardiovascular magnetic resonance and single-photon emission computed tomography for diagnosis of coronary heart disease (CE-MARC): a prospective trial. Lancet 2012; 379: 453-460.

8. Jahnke C, Nagel E, Gebker R, et al. Prognostic value of cardiac magnetic resonance stress tests: adenosine stress perfusion and dobutamine stress wall motion imaging. Circulation 2007; 115: 1769-1776.

9. Coelho-Filho OR, Seabra LF, Mongeon FP, et al. Stress myocardial perfusion imaging by CMR provides strong prognostic value to cardiac events regardless of patient's sex. JACC Cardiovasc Imaging 2011; 4: 850-861.

10. Moschetti K, Muzzarelli S, Pinget C, et al. Cost evaluation of cardiovascular magnetic resonance versus coronary angiography for the diagnostic work-up of coronary artery disease: application of the european cardiovascular magnetic resonance registry data to the German, United Kingdom, Swiss, and United States health care systems. J Cardiovasc Magn Reson 2012; 14: 35.

11. Pilz G, Patel PA, Fell U, et al. Adenosine-stress cardiac magnetic resonance imaging in suspected coronary artery disease: a net cost analysis and reimbursement implications. Int J Cardiovasc Imaging 2011; 27: 113-121.

12. Groothuis JGJ, Beek AM, Brinckman SL, et al. Low to intermediate probability of coronary artery disease: comparison of coronary CT angiography with first-pass MR myocardial perfusion imaging. Radiology 2010; 254: 384-392.

13. Groothuis JGJ, Beek AM, Meijerink MR, et al. Towards a noninvasive anatomical and functional diagnostic work-up of patients with suspected coronary artery disease. Neth Heart J 2010; 18: 270-273.

14. Lockie T, Ishida M, Perera D, et al. High-resolution magnetic resonance myocardial perfusion imaging at 3.0-Tesla to detect hemodynamically significant coronary stenoses as determined by fractional flow reserve. J Am Coll Cardiol 2011; 57: 70-75.

15. Jaarsma C, Leiner T, Bekkers SC, et al. Diagnostic performance of noninvasive myocardial perfusion imaging using single-photon emission computed tomography, cardiac magnetic resonance, and positron emission tomography imaging for the detection of obstructive coronary artery disease: a meta-analysis. J Am Coll Cardiol 2012; 59: 1719-1728. 